\title{
Relativistic Four-Component DFT Calculations of Vibrational Frequencies
}

\author{
Katarzyna Jakubowska, Magdalena Pecul,* and Kenneth Ruud
}

Cite This: J. Phys. Chem. A 2021, 125, 10315-10320

Read Online

ABSTRACT: We investigate the effect of relativity on harmonic vibrational frequencies. Density functional theory (DFT) calculations using the four-component Dirac-Coulomb Hamiltonian have been performed for 15 hydrides $\left(\mathrm{H}_{2} \mathrm{X}, \mathrm{X}=\mathrm{O}, \mathrm{S}, \mathrm{Se}, \mathrm{Te}, \mathrm{Po}\right.$; $\mathrm{XH}_{3}, \mathrm{X}=\mathrm{N}, \mathrm{P}, \mathrm{As}, \mathrm{Sb}, \mathrm{Bi}$; and $\mathrm{XH}_{4}, \mathrm{X}=\mathrm{C}, \mathrm{Si}, \mathrm{Ge}, \mathrm{Sn}, \mathrm{Pb}$ ) as well as for $\mathrm{HC} \equiv \mathrm{CPbH}_{3}$. The vibrational frequencies have been calculated using finite differences of the molecular energy with respect to geometrical distortions of the nuclei. The influences of the choice of basis set, exchange-correlation functional, and step length for the numerical differentiation on the calculated harmonic vibrational frequencies have been tested, and the method has been found to be numerically robust. Relativistic effects are noticeable

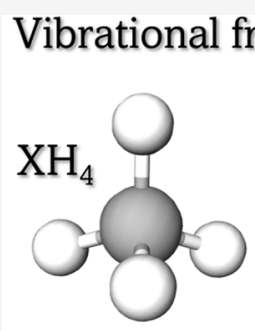

calculated with relativistic and nonrelativistic DFT method for the heavier congeners $\mathrm{H}_{2} \mathrm{Te}$ and $\mathrm{H}_{2} \mathrm{Po}, \mathrm{SbH}_{3}$ and $\mathrm{BiH}_{3}$, and $\mathrm{SnH}_{4}$ and $\mathrm{PbH}_{4}$ and are much more pronounced for the vibrational modes with higher frequencies. Spin-orbit effects constitute a very small fraction of the total relativistic effects, except for $\mathrm{H}_{2} \mathrm{Te}$ and $\mathrm{H}_{2}$ Po. For $\mathrm{HC} \equiv \mathrm{CPbH}_{3}$ we find that only the frequencies of the modes with large contributions from $\mathrm{Pb}$ displacements are significantly affected by relativity.

\section{INTRODUCTION}

For molecules containing heavy atoms, relativistic effects play a crucial role in their electronic structure and chemical bonding. ${ }^{1}$ Relativistic effects are commonly separated into scalar relativistic effects, which are due to (among other contributions) the mass-velocity and Darwin corrections, and the effects due to the spin-orbit interaction. The former lead for instance to contraction of the inner-shell orbitals (the energies of core levels are lower than those for the nonrelativistic case), and the latter result in the spin-orbit splitting of molecular orbital energy levels. Furthermore, the contraction of the inner-shell orbitals in turn increases the screening of the nuclear charge for the outer-shell electrons, giving rise to an indirect effect that results in expansion of the valence orbitals. These relativistic effects affect the valence orbitals involved with chemical bonding and consequently the potential energy surfaces. ${ }^{1}$

In most cases where potential energy surfaces are concerned, it is sufficient to account for scalar relativistic effects using for example effective core potentials, ${ }^{2}$ but for systems where strong spin-orbit effects may be expected, it is important to have an apparatus to calculate total relativistic effects using the four-component Dirac-Coulomb (or Dirac-Coulomb-Breit) Hamiltonian. Many four-component calculations have been carried out for dissociation energies ${ }^{1,3}$ and molecular gradients (first derivatives of the molecular energy with respect to distortions of the nuclei in the molecule $)^{4}$ as well as equilibrium geometries. ${ }^{4,5}$ In this contribution, we present the results of calculations of the molecular Hessian (second derivatives of the molecular energy with respect to nuclear distortions) and harmonic vibrational frequencies with the Dirac-Coulomb Hamiltonian. Four-component methods for the analytic calculation of molecular Hessians (and thus also harmonic vibrational frequencies) are currently not available in any computational chemistry program package. Instead, our calculations have been carried out with an external driver to the existing program package DiRAC. ${ }^{6}$ Test calculations have been performed for hydrides of elements from groups 14 $\left(\mathrm{XH}_{4}, \mathrm{X}=\mathrm{C}, \mathrm{Si}, \mathrm{Ge}, \mathrm{Sn}, \mathrm{Pb}\right), 15\left(\mathrm{XH}_{3}, \mathrm{X}=\mathrm{N}, \mathrm{P}, \mathrm{As}, \mathrm{Sb}, \mathrm{Bi}\right)$, and $16\left(\mathrm{H}_{2} \mathrm{X}, \mathrm{X}=\mathrm{O}, \mathrm{S}, \mathrm{Se}, \mathrm{Te}, \mathrm{Po}\right)$ and in addition for the acetylene derivative $\mathrm{HC} \equiv \mathrm{CPbH}_{3}$. Vibrational frequencies have been computed with the use of both relativistic and nonrelativistic methods in order to study the importance of the relativistic effects. Such calculations have previously been reported for halogen diatomics ${ }^{7}$ but not for polyatomic molecules.

There are well-established methods of calculating potential energy surfaces, including the molecular Hessian and vibra-

Received: August 20, 2021

Revised: November 15, 2021

Published: November 29, 2021 
tional frequencies, for molecular systems with substantial relativistic effects through the use of the zeroth-order regular approximation Hamiltonian, ${ }^{8,9}$ other two-component Hamiltonians, $^{10-13}$ and relativistic effective core potentials. ${ }^{2}$ In most cases these are sufficient for rendering the relativistic effects, apart from some systems with very strong spin-orbit coupling, such as some lanthanide compounds. ${ }^{1,14}$ However, a fourcomponent protocol will be useful for benchmarking more approximate treatments of relativistic effects. We have recently also demonstrated that the geometry dependence of NMR spin-spin coupling constants depends more strongly on relativistic effects than the spin-spin coupling constants themselves. ${ }^{15}$ This suggests that relativity may be important for zero-point vibrational (ZPV) corrections to NMR properties. For properties such as spin-spin coupling constants, a full relativistic treatment is necessary, and it is therefore important also to develop tools that allow vibrational frequencies to be calculated at the full four-component level of theory.

\section{METHODS}

Numerical Derivatives. Our program works as an external driver to the DIRAC program package. ${ }^{6}$ The method for calculating the molecular Hessian and thus also the harmonic vibrational frequencies is fully numerical. Computation of the Hessian is based on calculating the second derivatives of the molecular energy with respect to geometric distortions of the molecule using simple three-point formulas: ${ }^{16}$

$$
\begin{aligned}
& \frac{\mathrm{d}^{2} E}{\mathrm{~d} x_{\alpha}^{2}}=\frac{E_{x_{\alpha}+h}-2 E_{x_{\alpha}}+E_{x_{\alpha}-h}}{h^{2}} \\
& \frac{\mathrm{d}^{2} E}{\mathrm{~d} x_{\alpha} \mathrm{d} y_{\beta}}=\frac{E_{x_{\alpha}+h, y_{\beta}+h}-E_{x_{\alpha}-h, y_{\beta}+h}-E_{x_{\alpha}+h, y_{\beta}-h}+E_{x_{\alpha}-h, y_{\beta}-h}}{4 h^{2}}
\end{aligned}
$$

This means that the Hessian computation involves performing a number of energy calculations in which atoms are displaced from their original positions in all degrees of freedom. In the case of a molecule with $N$ atoms, $18 N^{2}+1$ single-energy computations need to be run to determine the full Hessian. Once the Hessian is obtained, the vibrational frequencies are calculated by diagonalization of the Hessian in its massweighted form.

When numerical differentiation is performed, it is important that an appropriate step length ( $h$ in the above equation) is used to ensure numerically accurate results. We performed test calculations of the vibrational frequencies for the water molecule with a number of different step lengths in the range of $10^{-1}-10^{-5} \AA$. The calculations turned out to be numerically stable for step lengths between $10^{-2}-10^{-4} \AA$. Similar test calculations for the $\mathrm{H}_{2}$ Po molecule revealed that in the case of heavier atoms, numerical stability shifts in the direction of larger step lengths $\left(5 \times 10^{-2}\right.$ to $\left.5 \times 10^{-3} \AA\right)$. All of the results can be found in the Supporting Information. On this basis, in all of the subsequent calculations we used a step length of $10^{-3} \AA$. The only exceptions were systems involving the heaviest atoms $(\mathrm{Pb}, \mathrm{Bi}$, and $\mathrm{Po})$, for which we used a step length of $10^{-2} \AA$. Using the differences between vibrational frequencies obtained with step lengths within the range of numerical stability, we were able to estimate the error bars to be about $3 \mathrm{~cm}^{-1}$.
In order to test the numerical stability of the three-point formula, additional calculations were carried out using a fivepoint formula: ${ }^{16}$

$$
\frac{\mathrm{d}^{2} E}{\mathrm{~d} x_{\alpha}{ }^{2}}=\frac{-E_{x_{\alpha}+2 h}+16 E_{x_{\alpha}+h}-30 E_{x_{\alpha}}+16 E_{x_{\alpha}-h}-E_{x_{\alpha}-2 h}}{12 h^{2}}
$$

No significant differences were found in comparison with the three-point formula with the same step length.

Geometry optimizations were performed using the DIRAC program $^{6}$ at the same level of theory as for the Hesssian calculations carried out afterward in order to ensure that the molecular gradient was equal to zero (a condition for the harmonic approximation). The convergence treshold for the gradient was $10^{-4}$.

Single-Energy Calculations. The four-component Dirac-Kohn-Sham (DKS) energy calculations were carried out with the DiRAC program. ${ }^{6}$ The uncontracted aug-cc-pVDZ basis $\operatorname{set}^{17}$ on the hydrogen atoms and Dyall's uncontracted triple- $\zeta$ basis set ${ }^{18-20}$ (dyall.v3z) on all of the other atoms were applied together with the B3LYP exchange-correlation functional, $^{21-24}$ unless stated otherwise.

For comparison, four-component calculations with spinorbit interactions switched off and nonrelativistic calculations were also carried out. In the case of the nonrelativistic computations, the speed of light was scaled to $2000.0 \mathrm{au}$ in the Dirac-Coulomb Hamiltonian, and in the case of the fourcomponent calculations without spin-orbit effects, the calculations were performed with Dyall's spin-free Hamiltonian $^{25}$ as implemented in Dirac.

Moreover, we carried out an investigation of the dependence of the results on the choice of exchange-correlation functional and basis set. In order to do so, four-component DKS calculations were performed using the PBE0 functional ${ }^{26}$ (to be compared to B3LYP) and also two additional basis sets: (1) the uncontracted aug-cc-pVDZ basis set ${ }^{17}$ on the hydrogen atoms and Dyall's uncontracted double- $\zeta$ basis set (dyall.v2z) (19,20 on all of the other atoms and (2) the uncontracted aug-cc-pVQZ basis set ${ }^{17}$ on the hydrogen atoms and Dyall's uncontracted quadrupole- $\zeta$ basis set (dyall.v4z) $)^{19,20}$ on all of the other atoms.

The convergence threshold for all of the single-energy calculations was $10^{-6}$.

Since an analytical method for calculating the molecular Hessian is implemented in the DALTON program, ${ }^{27,28}$ some calculations were performed with this program for comparison. We note that DALTON allows only one-component nonrelativistic DFT calculations. All of the DALTON computations were run with the same uncontracted basis set and exchangecorrelation functional as above and were carried out using the geometry optimized in DIRAC at the nonrelativistic level of theory (the same geometry as the one used for nonrelativistic numerical calculations of vibrational frequencies).

\section{RESULTS AND DISCUSSION}

In order to test the newly developed method for calculating harmonic vibrational frequencies, simple systems consisting of three, four, or five atoms have been chosen:

- $\mathrm{H}_{2} \mathrm{X}$ where $\mathrm{X}=\mathrm{O}, \mathrm{S}, \mathrm{Se}, \mathrm{Te}, \mathrm{Po}$;

- $\mathrm{XH}_{3}$ where $\mathrm{X}=\mathrm{N}, \mathrm{P}, \mathrm{As}, \mathrm{Sb}, \mathrm{Bi}$;

- $\mathrm{XH}_{4}$ where $\mathrm{X}=\mathrm{C}, \mathrm{Si}, \mathrm{Sn}, \mathrm{Pb}$. 
In addition to this, to illustrate the usefulness of the presented method for a larger system, we have calculated vibrational frequencies for an acetylene derivative, $\mathrm{HC} \equiv \mathrm{CPbH}_{3}$.

Influences of the Basis Set and Exchange-Correlation Functional on the Vibrational Frequencies. The results of four-component DFT calculations employing either the B3LYP or PBE0 functional for the vibrational frequencies of $\mathrm{H}_{2} \mathrm{X}(\mathrm{X}=\mathrm{O}, \mathrm{S}, \mathrm{Se}, \mathrm{Te}, \mathrm{Po})$ can be found in Table 1. In most

Table 1. Vibrational Frequencies for $\mathrm{H}_{2} \mathrm{X}$ : Comparison of Results Calculated with Either the B3LYP or PBE0 Functional $^{a}$

$\begin{array}{ccccc}\mathrm{X} & \text { functional } & \omega_{1}\left[\mathrm{~cm}^{-1}\right] & \omega_{2}\left[\mathrm{~cm}^{-1}\right] & \omega_{3}\left[\mathrm{~cm}^{-1}\right] \\ \mathrm{O} & \text { B3LYP } & 3918 & 3815 & 1623 \\ & \text { PBE0 } & 3983 & 3877 & 1630 \\ \mathrm{~S} & \text { B3LYP } & 2686 & 2671 & 1206 \\ & \text { PBE0 } & 2730 & 2714 & 1199 \\ \mathrm{Se} & \text { B3LYP } & 2401 & 2388 & 1061 \\ & \text { PBE0 } & 2448 & 2434 & 1059 \\ \mathrm{Te} & \text { B3LYP } & 2109 & 2102 & 885 \\ & \text { PBE0 } & 2152 & 2144 & 885 \\ \text { Po } & \text { B3LYP } & 1846 & 1829 & 777 \\ & \text { PBE0 } & 1901 & 1885 & 778\end{array}$

${ }^{a}$ Four-component DKS Hamiltonian with the indicated functional and the aug-cc-pVTZ (on H) + dyall.v3z (on X) basis set.

cases the frequencies obtained with PBE0 are larger, but the differences between the results obtained with PBEO and B3LYP do not exceed $3 \%$ in any case. Taking this into consideration, it seems that in this case B3LYP and PBE0 would produce comparable results. The B3LYP functional has been chosen for the following calculations because of its good performance for vibrational properties reported in the literature. $^{29-31}$

The results of the four-component DFT calculations of vibrational frequencies for $\mathrm{H}_{2} \mathrm{X}$ systems, carried out with double- $\zeta$, triple- $\zeta$, and quadruple- $\zeta$ quality basis sets, can be found in Table 2 . The differences between vibrational

Table 2. Vibrational Frequencies for $\mathrm{H}_{2} \mathrm{X}$ : Comparison of Results Calculated with Three Different Basis Sets ${ }^{a}$

$\begin{array}{ccccc}\mathrm{X} & \text { basis set } & \omega_{1}\left[\mathrm{~cm}^{-1}\right] & \omega_{2}\left[\mathrm{~cm}^{-1}\right] & \omega_{3}\left[\mathrm{~cm}^{-1}\right] \\ \mathrm{O} & \mathrm{DZ}^{b} & 3887 & 3787 & 1630 \\ & \mathrm{TZ}^{c} & 3918 & 3815 & 1623 \\ & \mathrm{QZ}^{d} & 3918 & 3816 & 1626 \\ \mathrm{~S} & \mathrm{DZ}^{b} & 2680 & 2666 & 1196 \\ & \mathrm{TZ}^{c} & 2686 & 2671 & 1206 \\ & \mathrm{QZ}^{d} & 2688 & 2675 & 1208 \\ \mathrm{Se} & \mathrm{DZ}^{b} & 2408 & 2393 & 1059 \\ & \mathrm{TZ}^{c} & 2401 & 2388 & 1061 \\ & \mathrm{QZ}^{d} & 2407 & 2394 & 1060 \\ \mathrm{Te} & \mathrm{DZ}^{b} & 2114 & 2107 & 890 \\ & \mathrm{TZ}^{c} & 2109 & 2102 & 885 \\ & \mathrm{QZ}^{d} & 2117 & 2110 & 885 \\ \mathrm{Po} & \mathrm{DZ}^{b} & 1844 & 1827 & 777 \\ & \mathrm{TZ}^{c} & 1846 & 1829 & 777 \\ & \mathrm{QZ}^{d} & 1848 & 1832 & 778\end{array}$

${ }^{a}$ Four-component DKS Hamiltonian with the B3LYP functional and the indicated basis set. ${ }^{b}$ aug-cc-pVDZ (on $\left.\mathrm{H}\right)+$ dyall.v2z (on X). ${ }^{c}$ aug-cc-pVTZ (on $\left.\mathrm{H}\right)+$ dyall.v3z (on X). ${ }^{d}$ aug-cc-pVQZ (on $\left.\mathrm{H}\right)+$ dyall.v4z (on $\mathrm{X}$ ). frequencies obtained with these three basis sets are almost negligible. The biggest differences occur between $\mathrm{DZ}$ and $\mathrm{QZ}$ for the $\mathrm{H}_{2} \mathrm{O}$ molecule, yet even in this case these differences are not larger than $1 \%$ of the values, being at most $31 \mathrm{~cm}^{-1}$. In all other cases, the differences do not exceed $10 \mathrm{~cm}^{-1}$. In light of the above findings, the triple- $\zeta$-quality basis set appears to be an optimal compromise between accuracy and computational cost, and this basis set has been used in all of the following calculations.

Numerical versus Analytical Hessian. As numerical methods for calculating the molecular Hessian will inevitably have limitations on the numerical accuracy, we have tried to estimate these by comparing the numerical harmonic vibrational frequencies with the results obtained with the analytical nonrelativistic method implemented in the DaLTON program. The comparison of the calculated harmonic vibrational frequencies can be found in Table 3 . We obtained excellent agreement in case of the $\mathrm{H}_{2} \mathrm{O}, \mathrm{H}_{2} \mathrm{~S}$ and $\mathrm{H}_{2} \mathrm{Se}$ molecules.

Table 3. Vibrational Frequencies for $\mathrm{H}_{2} \mathrm{X}$ : Comparison of Results Calculated with Relativistic and Nonrelativistic DFT Methods $^{a}$

\begin{tabular}{|c|c|c|c|c|c|}
\hline \multirow{2}{*}{$\begin{array}{l}X \\
O\end{array}$} & \multicolumn{2}{|c|}{ method $^{b}$} & \multirow{2}{*}{$\begin{array}{c}\omega_{1}\left[\mathrm{~cm}^{-1}\right] \\
\left(\mathrm{A}_{1} \text { symmetry, }\right. \\
\mathrm{X}-\mathrm{H} \\
\text { symmetric } \\
\text { stretch }) \\
3918\end{array}$} & \multirow{2}{*}{$\begin{array}{c}\omega_{2}\left[\mathrm{~cm}^{-1}\right] \\
\left(\mathrm{B}_{2} \text { symmetry, }\right. \\
\mathrm{X}-\mathrm{H} \\
\text { asymmetric } \\
\text { stretch }) \\
3815\end{array}$} & \multirow{2}{*}{$\begin{array}{c}\omega_{3}\left[\mathrm{~cm}^{-1}\right] \\
\left(A_{1} \text { symmetry, }\right. \\
\mathrm{H}-\mathrm{X}-\mathrm{H} \\
\text { bend }) \\
1623\end{array}$} \\
\hline & num & rel & & & \\
\hline & & no SO & 3902 & 3799 & 1627 \\
\hline & & nrel & 3920 & 3817 & 1623 \\
\hline & anal & nrel & 3920 & 3818 & 1623 \\
\hline & exper & ental $^{32}$ & 3756 & 3657 & 1595 \\
\hline \multirow[t]{5}{*}{ S } & num & rel & 2686 & 2671 & 1206 \\
\hline & & no SO & 2685 & 2671 & 1205 \\
\hline & & nrel & 2690 & 2676 & 1204 \\
\hline & anal & nrel & 2690 & 2676 & 1205 \\
\hline & exper & ental $^{32}$ & 2626 & 2615 & 1183 \\
\hline \multirow[t]{5}{*}{ Se } & num & rel & 2401 & 2388 & 1061 \\
\hline & & no SO & 2404 & 2390 & 1061 \\
\hline & & nrel & 2416 & 2404 & 1058 \\
\hline & anal & nrel & 2418 & 2406 & 1059 \\
\hline & exper & ental ${ }^{32}$ & 2358 & 2345 & 1034 \\
\hline \multirow[t]{5}{*}{$\mathrm{Te}$} & num & rel & 2109 & 2102 & 885 \\
\hline & & no SO & 2122 & 2115 & 888 \\
\hline & & nrel & 2147 & 2142 & 884 \\
\hline & anal & nrel & 2147 & 2142 & 885 \\
\hline & exper & ental $^{33}$ & 2072 & 2065 & 861 \\
\hline \multirow[t]{4}{*}{ Po } & num & rel & 1845 & 1828 & 775 \\
\hline & & no SO & 1977 & 1972 & 812 \\
\hline & & nrel & 2032 & 2031 & 806 \\
\hline & anal & nrel & 2032 & 2030 & 809 \\
\hline
\end{tabular}

${ }^{a}$ B3LYP functional, aug-cc-pVTZ (on $\left.\mathrm{H}\right)+$ dyall.v3z (on X) basis set. ${ }^{b}$ Fundamental vibrational frequencies are reported for the experimental data. For the calculated results, the following notation is used: num, numerical; anal, analytic; rel, relativistic; nrel, nonrelativistic; no SO, no spin-orbit coupling.

Influence of Relativity on the Vibrational Frequencies. Harmonic vibrational frequencues calculated with the relativistic and nonrelativistic methods are summarized in Tables 3 - 5. As can be noted, in almost all cases the relativistic vibrational frequencies are smaller than the corresponding nonrelativistic values, that is, relativity decreases 
Table 4. Vibrational Frequencies for $\mathrm{XH}_{3}$ : Comparison of Results Calculated with Relativistic and Nonrelativistic DFT Methods $^{a}$

\begin{tabular}{|c|c|c|c|c|c|}
\hline $\mathrm{X}$ & method $^{c}$ & $\begin{array}{c}\omega_{1}\left[\mathrm{~cm}^{-1}\right] A_{1} \text { symmetry, } \mathrm{X}-\mathrm{H} \\
\text { symmetric stretch }\end{array}$ & $\begin{array}{c}\omega_{2}\left[\mathrm{~cm}^{-1}\right] \text { (E symmetry, } \mathrm{X}-\mathrm{H} \\
\text { asymmetric stretch) }^{b}\end{array}$ & $\begin{array}{c}\omega_{3}\left[\mathrm{~cm}^{-1}\right](\text { E symmetry, } \mathrm{H}- \\
\mathrm{X}-\mathrm{H} \text { scissor })^{b}\end{array}$ & $\begin{array}{c}\omega_{4}\left[\mathrm{~cm}^{-1}\right]\left(\mathrm{A}_{1} \text { symmetry, }\right. \\
\mathrm{X}-\mathrm{H} \text { wag })\end{array}$ \\
\hline \multirow[t]{4}{*}{$\mathrm{N}$} & rel & 3596 & 3476 & 1661 & 1019 \\
\hline & no SO & 3584 & 3465 & 1663 & 1029 \\
\hline & nrel & 3587 & 3467 & 1662 & 1024 \\
\hline & experimental $^{32}$ & 3444 & 3337 & 1627 & 950 \\
\hline \multirow[t]{4}{*}{$\mathrm{P}$} & rel & 2385 & 2374 & 1136 & 1016 \\
\hline & no SO & 2385 & 2375 & 1136 & 1016 \\
\hline & nrel & 2389 & 2379 & 1136 & 1024 \\
\hline & experimental $^{32}$ & 2328 & 2323 & 1118 & 992 \\
\hline \multirow[t]{4}{*}{ As } & rel & 2168 & 2154 & 1016 & 937 \\
\hline & no $\mathrm{SO}$ & 2168 & 2154 & 1017 & 937 \\
\hline & nrel & 2183 & 2171 & 1016 & 930 \\
\hline & experimental $^{32}$ & 2123 & 2116 & 1003 & 906 \\
\hline \multirow[t]{4}{*}{$\mathrm{Sb}$} & rel & 1933 & 1926 & 844 & 812 \\
\hline & no $\mathrm{SO}$ & 1933 & 1926 & 842 & 809 \\
\hline & nrel & 1961 & 1958 & 842 & 803 \\
\hline & experimental $^{34}$ & 1894 & 1891 & 831 & 782 \\
\hline \multirow[t]{4}{*}{$\mathrm{Bi}$} & rel & 1768 & 1766 & 764 & 750 \\
\hline & no $\mathrm{SO}$ & 1796 & 1788 & 773 & 760 \\
\hline & nrel & 1855 & 1852 & 765 & 742 \\
\hline & experimental $1^{34}$ & 1734 & 1733 & 751 & 726 \\
\hline
\end{tabular}

${ }^{a}$ B3LYP functional, aug-cc-pVTZ (on H) + dyall.v3z (on X) basis set. ${ }^{b}$ No symmetry has been used, so frequencies of degenerate vibrations vary (by at most $2 \mathrm{~cm}^{-1}$ ). Arithmetic averages are given. ${ }^{c}$ Fundamental vibrational frequencies are reported for the experimental data. For the calculated results, the following notation is used: rel, relativistic; nrel, nonrelativistic; no SO, no spin-orbit coupling.

Table 5. Vibrational Frequencies for $\mathrm{XH}_{4}$ : Comparison of Results Calculated with Relativistic and Nonrelativistic DFT Methods $^{a}$

\begin{tabular}{|c|c|c|c|c|c|}
\hline $\mathrm{X}$ & method $^{c}$ & $\begin{array}{c}\omega_{1}\left[\mathrm{~cm}^{-1}\right]\left(\mathrm{A}_{1} \text { symmetry, } \mathrm{X}-\mathrm{H}\right. \\
\text { symmetric stretch }\end{array}$ & $\begin{array}{c}\omega_{2}\left[\mathrm{~cm}^{-1}\right]\left(\mathrm{T}_{2} \text { symmetry, } \mathrm{X}-\mathrm{H}\right. \\
\text { asymmetric stretch })\end{array}$ & $\begin{array}{c}\omega_{3}\left[\mathrm{~cm}^{-1}\right] \text { (E symmetry, } \\
\mathrm{H}-\mathrm{X}-\mathrm{H} \text { twist })^{b}\end{array}$ & $\begin{array}{c}\omega_{4}\left[\mathrm{~cm}^{-1}\right]\left(\mathrm{T}_{2} \text { symmetry, } \mathrm{H}-\right. \\
\mathrm{X}-\mathrm{H} \text { scissor })^{b}\end{array}$ \\
\hline \multirow[t]{4}{*}{$\mathrm{C}$} & rel & 3135 & 3032 & 1555 & 1337 \\
\hline & no SO & 3127 & 3025 & 1557 & 1339 \\
\hline & nrel & 3127 & 3025 & 1556 & 1339 \\
\hline & experimental $^{32}$ & 3019 & 2917 & 1534 & 1306 \\
\hline \multirow[t]{4}{*}{$\mathrm{Si}$} & rel & 2237 & 2227 & 977 & 918 \\
\hline & no SO & 2237 & 2227 & 977 & 918 \\
\hline & nrel & 2238 & 2228 & 976 & 917 \\
\hline & experimental $^{32}$ & 2191 & 2187 & 975 & 914 \\
\hline \multirow[t]{4}{*}{ Ge } & rel & 2144 & 2136 & 934 & 827 \\
\hline & no SO & 2143 & 2136 & 932 & 826 \\
\hline & nrel & 2144 & 2139 & 925 & 823 \\
\hline & experimental $^{32}$ & 2114 & 2106 & 931 & 819 \\
\hline \multirow[t]{3}{*}{ Sn } & rel & 1929 & 1927 & 753 & 686 \\
\hline & no SO & 1930 & 1927 & 752 & 684 \\
\hline & nrel & 1930 & 1926 & 737 & 678 \\
\hline \multirow[t]{3}{*}{$\mathrm{Pb}$} & rel & 1839 & 1827 & 686 & 609 \\
\hline & no SO & 1847 & 1823 & 693 & 616 \\
\hline & nrel & 1847 & 1839 & 664 & 609 \\
\hline
\end{tabular}

${ }^{a}$ B3LYP functional, aug-cc-pVTZ (on H) + dyall.v3z (on X) basis set. ${ }^{b}$ No symmetry has been used, so frequencies of degenerate vibrations vary (by at most $2 \mathrm{~cm}^{-1}$ ). Arithmetic averages are given. ${ }^{c}$ Fundamental vibrational frequencies are reported for the experimental data. For the calculated results, the following notation is used: rel, relativistic; nrel, nonrelativistic; no SO, no spin-orbit coupling.

the bond strength. In the case of the $\mathrm{H}_{2} \mathrm{X}$ systems, relativistic effects are significant for $\mathrm{H}_{2} \mathrm{Te}\left(2 \%\right.$ for $\omega_{1}$ and $\left.\omega_{2}\right)$ and $\mathrm{H}_{2} \mathrm{Po}$ $\left(10 \%\right.$ for $\omega_{1}$ and $\omega_{2}, 3 \%$ for $\left.\omega_{3}\right)$. Also in the case of the $\mathrm{XH}_{3}$ molecules, relativistic effects are not negligible for the two heaviest congeners, $2 \%$ for $\omega_{1}$ and $\omega_{2}$ in $\mathrm{SbH}_{3}$ and $8 \%$ for $\omega_{1}$, $5 \%$ for $\omega_{2}$, and $2 \%$ for $\omega_{3}$ in $\mathrm{BiH}_{3}$. In the case of the $\mathrm{XH}_{4}$ molecules, relativistic effects are negligible for all but one mode, $\omega_{3}$ (E symmetry mode), for $\mathrm{SnH}_{4}(2 \%)$ and $\mathrm{PbH}_{4}(6 \%)$.
It should be stressed here that the precentage change in the values when calculated with relativistic and nonrelativistic methods varies for each vibrational mode.

In addition, for all of the molecules, relativistic fourcomponent calculations without spin-orbit effects have been performed (Table 4). These results show that in the case of $\mathrm{XH}_{3}$, all of the relativistic effects are in fact scalar relativistic effects, whereas in the case of $\mathrm{H}_{2} \mathrm{X}$, spin-orbit effects play a 
crucial role. Spin-orbit effects constitute about $30 \%$ of the relativistic effects in the case of $\mathrm{H}_{2} \mathrm{Te}$ and as much as $70 \%$ in the case of $\mathrm{H}_{2} \mathrm{Po}$.

Comparison with Experimental Values. When comparing the results obtained with the experimental values, one should keep in mind that the diagonalization of the molecular Hessian gives us harmonic vibrational frequencies. Thus, anharmonicity is not taken into account, and this will lead to some difference between the results and the experimental values. In our case, the differences do not exceed 5\%, in line with the expected magnitude of anharmonic corrections. ${ }^{35}$

Vibrational Frequencies for $\mathrm{HC} \equiv \mathrm{CPbH}_{3}$. To illustrate the usefulness of the presented method and to study the effects of relativity on vibrational frequencies for a more complex system where only some of the modes involve the heavy atom, we have calculated the vibrational frequencies for the acetylene derivative $\mathrm{HC} \equiv \mathrm{CPbH}_{3}$ with both relativistic and nonrelativistic approaches. The motivation for choosing this particular system was our previous work, ${ }^{15}$ where we showed that for this molecule the relativistic effects on the derivatives of the indirect spin-spin coupling constants with respect to molecular geometry parameters tend to be more pronounced than the effects on the coupling constants themselves. The ZPV corrections calculated at the nonrelativistic level are therefore not necessarily reliable. The uncontracted aug-ccpVDZ basis set ${ }^{17}$ was used on the hydrogen and carbon atoms and Dyall's uncontracted triple- $\zeta$ basis set $(\text { dyall.v3z })^{18-20}$ on the lead atom have been applied together with the B3LYP exchange-correlation functional. ${ }^{21-24}$ The results are collected in Table 6.

Table 6. Vibrational Frequencies for $\mathrm{HC} \equiv \mathrm{CPbH}_{3}$ : Comparison of Results Calculated with Relativistic and Nonrelativistic DFT Methods ${ }^{a}$

\begin{tabular}{lcc}
\multicolumn{1}{c}{ mode } & $\begin{array}{c}\text { relativistic } \\
{\left[\mathrm{cm}^{-1}\right]}\end{array}$ & $\begin{array}{c}\text { nonrelativistic } \\
{\left[\mathrm{cm}^{-1}\right]}\end{array}$ \\
$\mathrm{C}-\mathrm{H}$ stretch $\left(\mathrm{A}_{1}\right.$ symmetry) & 3445 & 3440 \\
$\mathrm{C}-\mathrm{C}$ stretch $\left(\mathrm{A}_{1}\right.$ symmetry) & 2117 & 2116 \\
$\mathrm{~Pb}-\mathrm{H}$ asymmetric stretch & 1857 & 1840 \\
$\quad$ E symmetry $)^{b}$ & 1846 & 1846 \\
$\mathrm{~Pb}-\mathrm{H}$ symmetric stretch $\left(\mathrm{A}_{1}\right.$ & & \\
symmetry) & 708 & 702 \\
$\mathrm{C}-\mathrm{C}-\mathrm{H}$ bend $\left(\mathrm{A}_{2} \text { symmetry }\right)^{b}$ & 621 & 613 \\
$\mathrm{H}-\mathrm{Pb}-\mathrm{H}$ wag $\left(\mathrm{A}_{1}\right.$ symmetry) & 641 & 597 \\
$\mathrm{H}-\mathrm{Pb}-\mathrm{H}$ scissor $(\mathrm{E} \text { symmetry })^{b}$ & 482 & 429 \\
$\mathrm{H}-\mathrm{C}-\mathrm{C}-\mathrm{Pb}$ wag $\left(\mathrm{A}_{2} \text { symmetry }\right)^{b}$ & 384 & 409 \\
$\mathrm{C}-\mathrm{Pb}$ stretch $\left(\mathrm{A}_{1}\right.$ symmetry) & 187 & 145 \\
$\mathrm{C}-\mathrm{C}-\mathrm{Pb}$ bend $\left(\mathrm{A}_{2} \text { symmetry }\right)^{b}$ &
\end{tabular}

${ }^{a}$ B3LYP functional, aug-cc-pVTZ (on $\mathrm{H}$ and $\mathrm{C}$ ) + dyall.v3z (on $\mathrm{Pb}$ ) basis set. ${ }^{b}$ No symmetry has been used, so frequencies of degenerate vibrations vary (by at most $5 \mathrm{~cm}^{-1}$ ). Arithmetic averages are given.

In the case of the $\mathrm{HC} \equiv \mathrm{CPbH}_{3}$ molecule, it seems that only vibrations that involve the $\mathrm{Pb}$ atom are significantly affected by relativity. There is almost no difference between the relativistic and nonrelativistic values of vibrational frequencies for $\mathrm{C}-\mathrm{H}$ stretching, $\mathrm{C}-\mathrm{C}$ stretching, and $\mathrm{C}-\mathrm{C}-\mathrm{H}$ bending. This finding may be useful for future calculations of vibrational effects on molecular properties for large molecules, since it may allow for relativity to be taken into account only for selected localized modes. Similar findings were previously reported by Berger et al. $^{36}$ In addition to this, the relativistic effects are much more pronounced for deformation modes than for stretching modes. The difference between the vibrational frequencies calculated with relativistic and nonrelativistic methods for the $\mathrm{C}-\mathrm{C}-\mathrm{Pb}$ bend exceeds $20 \%$ of the value, whereas for the $\mathrm{C}-\mathrm{Pb}$ stretch it is only little more than $5 \%$.

\section{CONCLUSIONS}

We have presented a numerical method for calculating the molecular Hessian and harmonic vibrational frequencies with relativistic four-component DFT. Test calculations have been performed for hydrides of elements from groups 14, 15, and 16. We have achieved good agreement with an analytical nonrelativistic DFT method.

Relativistic effects become significant primarily for the hydrides containing atoms from the fifth and sixth rows of the periodic table and are much more pronounced for the vibrational modes with higher frequencies. Spin-orbit effects constitute a very small fraction of the relativistic effects on the whole, with the exception of $\mathrm{H}_{2} \mathrm{Te}$ and $\mathrm{H}_{2} \mathrm{Po}$. Additional calculations for $\mathrm{HC} \equiv \mathrm{CPbH}_{3}$ show that only the frequencies of the modes with large contributions from $\mathrm{Pb}$ displacements are significantly affected by relativity.

This work is considered a stepping stone towards the development of a four-component relativistic numerical method for calculating ZPV corrections to NMR parameters (spin-spin coupling constants and shielding constants).

\section{ASSOCIATED CONTENT}

\section{SI Supporting Information}

The Supporting Information is available free of charge at https://pubs.acs.org/doi/10.1021/acs.jpca.1c07398.

Vibrational frequencies for $\mathrm{H}_{2} \mathrm{O}$ and $\mathrm{H}_{2} \mathrm{Po}$ obtained with different step lengths and different optimized geometries (PDF)

\section{AUTHOR INFORMATION}

\section{Corresponding Author}

Magdalena Pecul - Faculty of Chemistry, University of Warsaw, 02-093 Warsaw, Poland; orcid.org/0000-00024872-9031; Email: mpecul@chem.uw.edu.pl

\section{Authors}

Katarzyna Jakubowska - Faculty of Chemistry, University of Warsaw, 02-093 Warsaw, Poland; 다이.org/0000-00031607-0102

Kenneth Ruud - Hylleraas Centre for Quantum Molecular Sciences, Department of Chemistry, UiT - The Arctic University of Norway, N-9019 Tromsø, Norway; (1) orcid.org/0000-0003-1006-8482

Complete contact information is available at: https://pubs.acs.org/10.1021/acs.jpca.1c07398

Notes

The authors declare no competing financial interest.

\section{ACKNOWLEDGMENTS}

K.J. acknowledges financial support from the Polish National Science Centre on the basis of the decision DEC-2019/33/N/ ST4/01691. K.R. acknowledges support from the Research Council of Norway through a Centre of Excellence Grant (Grant 262695). 


\section{REFERENCES}

(1) Pyykko, P. Relativistic effects in structural chemistry. Chem. Rev. 1988, 88, 563-594.

(2) Chen, H.; Krasowski, M.; Fitzgerald, G. Density functional pseudopotential studies of molecular geometries, vibrations, and binding energies. J. Chem. Phys. 1993, 98, 8710.

(3) Malli, G. Ab initio all-electron fully relativistic Dirac-Fock selfconsistent field calculations for UCI6. Mol. Phys. 2003, 101, 287-294.

(4) Di Remigio, R.; Bast, R.; Frediani, L.; Saue, T. Four-Component Relativistic Calculations in Solution with the Polarizable Continuum Model of Solvation: Theory, Implementation, and Application to the Group 16 Dihydrides $\mathrm{H}_{2} \mathrm{X}(\mathrm{X}=\mathrm{O}, \mathrm{S}, \mathrm{Se}, \mathrm{Te}, \mathrm{Po})$. J. Phys. Chem. A 2015, 119, 5061-5077.

(5) Dohn, A. O.; Moller, K. B.; Sauer, S. P. A. Optimizing the Structure of Tetracyanoplatinate (II): A Comparison of Relativistic Density Functional Theory Methods. Curr. Inorg. Chem. 2013, 3, 213-219.

(6) Saue, T.; Visscher, L.; Jensen, H. J. Aa.; Bast, R.; Bakken, V.; Dyall, K. G.; Dubillard, S.; Ekström, U.; Eliav, E.; Enevoldsen, T.; et al., DIRAC, a Relativistic Ab Initio Electronic Structure Program, DIRAC18 release, 2018 (available at https://doi.org/10.5281/ zenodo.2253986; also see http://www.diracprogram.org).

(7) Fossgaard, O.; Gropen, O.; Corral Valero, M.; Saue, T. On the performance of four-component relativistic density functional theory: Spectroscopic constants and dipole moments of the diatomics HX and $\mathrm{XY}(\mathrm{X}, \mathrm{Y}=\mathrm{F}, \mathrm{Cl}, \mathrm{Br}$, and I). J. Chem. Phys. 2003, 118, 1041810430.

(8) van Lenthe, E.; Snijders, J. G.; Baerends, E. J. The zero-order regular approximation for relativistic effects: The effect of spin-orbit coupling in closed shell molecules. J. Chem. Phys. 1996, 105, 65056516.

(9) van Lenthe, J.-H.; van Lingen, J. N. J. Note on the Calculation of Analytical Hessians in the Zeroth-Order Regular Approximation (ZORA). Int. J. Quantum Chem. 2006, 106, 2525-2528.

(10) de Jong, W. A.; Harrison, R. J.; Dixon, D. A. Parallel DouglasKroll energy and gradients in NWChem: Estimating scalar relativistic effects using Douglas-Kroll contracted basis sets. J. Chem. Phys. 2001, $114,48$.

(11) Mayer, M.; Krüger, S.; Rösch, N. A two-component variant of the Douglas-Kroll relativistic linear combination of Gaussian-type orbitals density-functional method: Spin-orbit effects in atoms and diatomics. J. Chem. Phys. 2001, 115, 4411.

(12) Matveev, A.; Nasluzov, V.; Rösch, N. Linear response formalism for the Douglas-Kroll-Hess approach to the DiracKohn-Sham problem: First- and second-order nuclear displacement derivatives of the energy. Int. J. Quantum Chem. 2007, 107, 32363249.

(13) Vasiliu, M.; Peterson, K. A.; Dixon, D. A. Bond Dissociation Energies in Heavy Element Chalcogen and Halogen Small Molecules. J. Phys. Chem. A 2021, 125, 1892-1902.

(14) Solomonik, V. G.; Smirnov, A. N. Toward Chemical Accuracy in ab Initio Thermochemistry and Spectroscopy of Lanthanide Compounds: Assessing Core-Valence Correlation, Second-Order Spin-Orbit Coupling, and Higher Order Effects in Lanthanide Diatomics. J. Chem. Theory Comput. 2017, 13, 5240-5254.

(15) Jakubowska, K.; Pecul, M.; Jaszuński, M. Spin-spin coupling constants in $\mathrm{HC} \equiv \mathrm{CXH}_{3}$ molecules; $\mathrm{X}=\mathrm{C}, \mathrm{Si}, \mathrm{Ge}, \mathrm{Sn}$ and $\mathrm{Pb}$. Theor. Chem. Acc. 2018, 137, No. 41.

(16) Rottmann, K. Mathematische Formelsammlung; Springer Spectrum: Berlin, 1991.

(17) Dunning, T. H. Gaussian basis sets for use in correlated molecular calculations. I. The atoms boron through neon and hydrogen. J. Chem. Phys. 1989, 90, 1007-1023.

(18) Dyall, K. G. Relativistic and nonrelativistic finite nucleus optimized triple zeta basis sets for the $4 \mathrm{p}, 5 \mathrm{p}$ and $6 \mathrm{p}$ elements. Theor. Chem. Acc. 2002, 108, 335-340. The basis sets are available from the Dirac website at http://dirac.chem.sdu.dk/basisarchives/dyall/index. html.
(19) Dyall, K. G. Relativistic Quadruple-Zeta and Revised TripleZeta and Double-Zeta Basis Sets for the 4p, 5p, and 6p Elements. Theor. Chem. Acc. 2006, 115, 441. The basis sets are available from the Dirac website at http://dirac.chem.sdu.dk/basisarchives/dyall/ index.html.

(20) Dyall, K. G. Relativistic double-zeta, triple-zeta, and quadruplezeta basis sets for the light elements H-Ar. Theor. Chem. Acc. 2016, $135,128$.

(21) Lee, C.; Yang, W.; Parr, R. G. Development of the ColleSalvetti correlation-energy formula into a functional of the electron density. Phys. Rev. B: Condens. Matter Mater. Phys. 1988, 37, 785-789.

(22) Becke, A. D. Density-functional thermochemistry. III. The role of exact exchange. J. Chem. Phys. 1993, 98, 5648-5652.

(23) Vosko, S. H.; Wilk, L.; Nusair, M. Accurate spin-dependent electron liquid correlation energies for local spin density calculations: a critical analysis. Can. J. Phys. 1980, 58, 1200-1211.

(24) Stephens, P. J.; Devlin, F. J.; Chabalowski, C. F.; Frisch, M. J. $\mathrm{Ab}$ Initio Calculation of Vibrational Absorption and Circular Dichroism Spectra Using Density Functional Force Fields. J. Phys. Chem. 1994, 98, 11623-11627.

(25) Dyall, K. G. An exact separation of the spin-free and spindependent terms of the Dirac-Coulomb-Breit Hamiltonian. J. Chem. Phys. 1994, 100, 2118-2127.

(26) Adamo, C.; Barone, V. Toward reliable density functional methods without adjustable parameters: The PBE0 model. J. Chem. Phys. 1999, 110, 6158-6170.

(27) DALTON, a Molecular Electronic Structure Program, Dalton2020 release, 2020 (see http://daltonprogram.org/).

(28) Aidas, K.; Angeli, C.; Bak, K. L.; Bakken, V.; Bast, R.; Boman, L.; Christiansen, O.; Cimiraglia, R.; Coriani, S.; Dahle, P.; et al. The Dalton quantum chemistry program system. Wiley Interdiscip. Rev.: Comput. Mol. Sci. 2014, 4, 269-284.

(29) Scott, A. P.; Radom, L. Harmonic Vibrational Frequencies: An Evaluation of Hartree-Fock, Møller-Plesset, Quadratic Configuration Interaction, Density Functional Theory, and Semiempirical Scale Factors. J. Phys. Chem. 1996, 100, 16502-16513.

(30) Finley, J.; Stephens, P. Density functional theory calculations of molecular structures and harmonic vibrational frequencies using hybrid density functionals. J. Mol. Struct.: THEOCHEM 1995, 357, $225-235$.

(31) Carbonniere, P.; Lucca, T.; Pouchan, C.; Rega, N.; Barone, V. Vibrational computations beyond the harmonic approximation: Performances of the B3LYP density functional for semirigid molecules. J. Comput. Chem. 2005, 26, 384-388.

(32) Shimanouchi, T. Molecular Vibrational Frequencies. In NIST Chemistry WebBook; Linstrom, P. J., Mallard, W. G., Eds.; NIST Standard Reference Database Number 69; National Institute of Standards and Technology: Gaithersburg, MD.

(33) Flaud, J.-M.; Betrencourt, M.; Arcas, P.; Bürger, H.; Polanz, O.; Lafferty, W. Simultaneous Analysis of the 2v2, v1, and v3 bands of Hydrogen Telluride. J. Mol. Spectrosc. 1997, 182, 396-420.

(34) Jacox, M. E. Vibrational and Electronic Energy Levels of Polyatomic Transient Molecules. In NIST Chemistry WebBook; Linstrom, P. J., Mallard, W. G., Eds.; NIST Standard Reference Database Number 69; National Institute of Standards and Technology: Gaithersburg, MD.

(35) Ringholm, M.; Jonsson, D.; Bast, R.; Gao, B.; Thorvaldsen, A. J.; Ekström, U.; Helgaker, T.; Ruud, K. Analytic cubic and quartic force fields using density-functional theory. J. Chem. Phys. 2014, 140, 034103.

(36) Berger, R. J. F.; Rettenwander, D.; Spirk, S.; Wolf, C.; Patzschke, M.; Ertl, M.; Monkowius, U.; Mitzel, N. W. Relativistic effects in triphenylbismuth and their influence on molecular structure and spectroscopic properties. Phys. Chem. Chem. Phys. 2012, 14, $15520-15524$. 